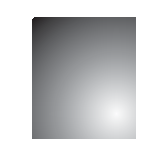

\title{
As Reações dos Consumidores diante das Falhas de Serviços a Partir da TeOria da Atribuição
}

\author{
The Reactions Consumer Services Before the Failures from the \\ Attribution Theory
}

\section{Francisca Flávia Plutarco Lopes}

Mestre em Administração pela Universidade Estadual do Ceará. Professora da Universidade de Fortaleza, UNIFOR e das Faculdades Cearenses. Fortaleza, CE. Brasil.E-mail: flaviaplutarco@hotmail.com

\section{Márcio de Oliveira Mota}

Doutor em Administração de Empresas pela Universidade de Fortaleza. Professor do Mestrado Acadêmico em Administração, Universidade Estadual do Ceará, UECE. Fortaleza, CE. Brasil.E-mail: marcio@marciomota.com

\section{Ana Augusta Ferreira de Freitas}

Doutora em Engenharia da Produção pela Universidade Federal de Santa Catarina. Professora do Mestrado Acadêmico em Administração, Universidade Estadual do Ceará, UECE. Fortaleza, CE. Brasil. Email: ana.freitas@uece.br

\section{Resumo}

Este estudo foi desenvolvido a fim de compreender as razões das rupturas das relações comerciais, a maneira como ela ocorre, as emoções relacionadas e a forma como os consumidores estão dispostos a reiniciar suas relações. A estrutura teórica utilizada para compreender esses processos foi a da Teoria da Atribuição, que examina as reações dos consumidores em falhas de serviço. Em consonância com os objetivos desta pesquisa, o modelo conceitual proposto foi testado em um estudo de campo envolvendo 245 consumidores. Os dados coletados foram submetidos a testes estatísticos por meio de modelos regressivos e modelagem em equações estruturais, no intuito de testar o modelo teórico proposto. O estudo evidencia um novo campo para explorar na literatura de marketing relacionado à retenção de clientes, baseado na reversão dos sentimentos de insatisfação que são os motivos antecedentes que levam os consumidores a romperem ou a restabelecerem seus relacionamentos com os provedores de serviços.

Palavras-chave: Dissolução de Relações Comerciais. Teoria da Atribuição. Marketing de Serviços.

\section{Abstract}

This study proposes to understand the reasons of the commercial ruptures, the way they occur, the emotions involved, and how consumers are willing to renew their relationships with the services providers. The conceptual background used to understand these processes was based on the Attribution Theory, which conception examines consumer reactions in service failures. The research design tested a proposed model by a field study involving 245 consumers. We used hierarchical multiple regressions and structural equation modeling in order to test our hypotheses. Our study also evidenced a new field to explore in the marketing literature related to customer retention based on the reversion of dissatisfaction feelings. They are the previous motives that lead consumers to break up or to re-establish their relationships with the service providers.

Keywords: Dissolution of Commercial Relationship. Attribution Theory. Services Marketing. 


\section{INTRODUÇÃO}

Compreender as razões dos consumidores em romperem suas relações com as organizações empresariais tem sido motivação para vários pesquisadores (BITNER; BOOMS; TETREAULT, 1990; COULTER; LIGAS, 2000). Tähtinen e Havila (2004), por exemplo, afirmam que não foi dada muita atenção ao tema até a década de 1990, principalmente no que diz respeito à compreensão de como, o porquê e quando essa relação terminou. Segundo os autores, o foco principal ainda está relacionado com a compreensão das razões dessa ruptura e seus antecedentes. Assim, o processo das rupturas e seus consequentes ainda são pouco discutidos na academia, apesar de sua relevância para os impactos de gestão de marketing. Em razão disso, Gedeon, Fearne e Poole (2009) sugerem que esse entendimento ainda é um desafio para pesquisadores, principalmente no que diz respeito à geração de evidências empíricas.

A fim de compreender as razões das rupturas das relações comerciais, a forma como ela ocorre, as emoções relacionadas e a forma como os consumidores estão dispostos a reiniciar suas relações, este estudo foi desenvolvido. Como arcabouço teórico subjacente, utilizou-se a Teoria da Atribuição (HEIDER, 1958; KELLEY, 1976; JONES et al., 1971) como uma estrutura útil para o exame das reações dos consumidores à falhas de serviço (WEINER, 2000). A base dessa teoria está presente no cotidiano dos consumidores em situações em que eles tendem a refletir sobre a origem de um problema em dimensões, ou seja, a causa e a responsabilidade, para formar opiniões que geram consequências e influências em decisões futuras.

Dessa forma, pode ser traçado um paralelo entre as ideias de Iglesias (2009) e a atribuição na finalização de relacionamentos comerciais, visto que em ambos os casos, a forma como o consumidor faz atribuições, a partir de uma percepção de falha na prestação do serviço, modifica as consequências do processo. $\mathrm{Na}$ finalização, o consumidor percebe a falha e busca uma resposta que pode ser atribuída a várias razões. Ao deduzir uma causa, esse entendimento do motivo da falha pode ser mediado por emoções e tem efeitos diretos no desejo do consumidor de voltar a comprar o produto ou adquirir o serviço (FOLKES; KOLETSKY; GRAHAM, 1987). Esse efeito é, até o momento, desconhecido. Nessa lacuna do conhecimento esta pesquisa se insere com objetivo de analisar a predisposição do consumidor ao retorno a um relacionamento comercial finalizado, a partir do processo atribucional, no contexto de serviços.

\section{Referencial Teórico}

O embasamento teórico deste trabalho encontra-se dividido em duas seções. Inicialmente trata-se do marketing de relacionamento e, de maneira específica, da fase final do ciclo de desenvolvimento de um relacionamento comercial: a sua finalização. Posteriormente, explora-se a relação entre a Teoria da Atribuição e a tomada de decisão no consumo, mostrando de que forma este arcabouço teórico pode ajudar na compreensão das finalizações de vínculos comerciais.

\subsection{Marketing de Relacionamento e a Finalização de Relacionamentos Comerciais}

O termo marketing de relacionamento foi usado pela primeira vez por Berry (1983), que o definiu como todos os serviços organizacionais de uma empresa que visam a atrair, manter e conquistar o cliente. $\mathrm{Na}$ ocasião, o autor identificava que a atração de clientes é apenas um processo intermediário no processo do marketing, pois o foco central do marketing é transformar clientes eventuais em clientes fiéis, solidificando um relacionamento mútuo. Esta nova concepção filosófica do marketing foi compartilhada por outros autores como Grönroos (1994), Morgan e Hunt (1994) e Robicheaux e Coleman (1995).

Tomando o marketing de relacionamento como um novo paradigma, e lançando luz na forma como os vínculos comerciais são formados e evoluem, Dwyer, Schurr e Oh (1987) avançaram ao proporem um modelo de desenvolvimento de relacionamentos entre empresas e consumidores. Ao indicarem a finalização como uma fase final, os autores alertam para a necessidade de estudar este processo como uma hipótese contrária ao relacionamento, não sendo possível entendê-lo de forma simplista. De fato, estudos posteriores que se basearam neste modelo mostraram que diversos fatores e elementos podem motivar a finalização de relacionamentos estabelecidos na esfera comercial. (e.g. ROOS; STRANDVIK, 1997; PRESSEY; MATHEWS, 2003) 
De maneira geral, entende-se que nos relacionamentos comerciais existem benefícios e, muitas vezes, durante a relação, existem problemas e dificuldades que em alguns casos a levam ao fim (TÄHTINEN, 2001). Embora seja um tópico importante e mais evidenciado nos últimos anos, Pressey e Selassie (2007) afirmam que a dissolução de relacionamentos ainda é um tema negligenciado em estudos na área de marketing. A finalização de um relacionamento comercial, na visão de Stewart (1998), tem sido descrita como um acontecimento econômico no qual um consumidor decide deixar de ser cliente de um fornecedor em particular. No entanto, conforme Halinen e Tähtinen (2000), essa finalização deveria ser entendida como um processo que é composto de percepções negativas, sentimentos desconfortáveis e reclamações. Nesta linha, Michalski (2004) definiu a dissolução de um relacionamento pelo consumidor como um processo de decisão de deixar ou sair de um relacionamento de negócios e não um acontecimento pontual e discreto.

Nesta mesma linha de argumentação, Roos e Strandvik (1997) e Michalski (2004) argumentam que a dissolução de relacionamentos comerciais ocorre por processos diferentes e pode variar de acordo com a sua duração e intensidade. A partir de estudos empíricos sobre o assunto, Roos e Strandvik (1997) observaram que a saída do relacionamento comercial pode ocorrer como um longo processo ou de maneira espontânea, além de poder ter uma resistência do consumidor em retornar ao provedor ou uma antecipação de um provável retorno. (COULTER; LIGAS, 2000)

Conhecer os meandros deste processo é de extrema utilidade para as empresas que, segundo Michalski (2004), podem prever sinais de insatisfação e a intenção do consumidor em finalizar o relacionamento, $e$ eventualmente reverter um possível fim. Como esteio teórico para entender melhor este assunto, a Teoria da Atribuição surge para auxiliar no entendimento das reações dos consumidores às falhas e suas consequências. A seção seguinte explora esse assunto.

\subsection{A Teoria da Atribuição nos Estudos sobre Consumo}

Uma pessoa busca explicar as causas de certos eventos ao procurar por razões da sua ocorrência. A Teoria da Atribuição, conforme Mizerki, Golden e
Kernan (1979), é composta por uma evolução de teorias que formam um campo de conhecimento na área de atribuição causal. Muitos estudos se baseiam nesta Teoria para analisar os sentimentos e as emoções que permeiam o processo de atribuição causal e as suas consequências afetivas. Isto porque, a compreensão das causas no processo tem um importante papel no comportamento das pessoas, fornecendo impulsos para ação e para decisões alternativas (BURNKRANT, 1975; WEINER, 2000), incluindo a esfera do consumo.

Sobre esse assunto, Folkes, Koetsky e Graham (1988) relatam que o estudo das inferências causais, em um relacionamento de consumo, colabora para a percepção de causa e efeito no processo atribucional e influencia no comportamento do consumidor. Bonifield e Cole (2007) explicam que depois de uma falha no serviço, os consumidores fazem julgamentos sobre as características da falha, buscando suas causas e a responsabilidade e daí derivando as consequências para o relacionamento comercial.

As dimensões causais e a responsabilidade são tratadas pela Teoria da Atribuição. Segundo Curren e Foles (1987), as causas podem ser estáveis ou instáveis, o que significa que o consumidor busca entender se a origem da falha é temporária ou permanente. Em termos de responsabilidade, segundo Huang (2008), que é também avaliada pelo consumidor como interna ou externa. Será interna quando o consumidor entender que colaborou com o fato negativo; e externa quando a percepção for de que a organização per se causou o fato negativo. Ao fazer reflexões quanto à responsabilidade da falha, o consumidor pode ter percepções diferentes quanto à responsabilidade da empresa pela falha. (MAGNINI et al., 2007)

Em termos de consequência, a percepção sobre a causa e responsabilidade pode despertar um desejo por recomendar uma empresa ou de rejeitá-la, esperando, por exemplo, um reembolso monetário. Pode acontecer ainda que o consumidor não altere as intenções e somente espere uma troca, ou que ele sinta o desejo de prejudicar a empresa. Tais dimensões são percebidas como dimensões independentes e em estudos sobre as reações dos consumidores quanto às falhas, à reação da empresa e às reações geradas pelas emoções, como raiva ou culpa, podem influenciar no comportamento do consumidor e nas intenções futuras quanto a 
comprar novamente daquela empresa/prestador de serviços. (FOLKES, 1987; WEINER, 2000)

A predisposição a retornar ao relacionamento comercial é concebida como sendo originada das reações e julgamentos do consumidor sobre sua intenção de comprar novamente com uma determinada empresa/prestador de serviços e a satisfação, levando em consideração a sua situação atual, a recordação das múltiplas transações comerciais vivenciadas e as expectativas futuras quanto à performance da empresa (HELLIER et al., 2002; MAGNINI et al., 2007). Sobre esse assunto, os estudos de Gilly e Gelb (1982) evidenciam que, em casos de insatisfação, o consumidor pode decidir por reclamar. A reação da empresa a essa reclamação pode ser condizente com as expectativas do consumidor.

O gerenciamento das reclamações mostrou-se como uma ferramenta poderosa para trabalhar de maneira positiva as intenções de retorno do consumidor ao relacionamento. Alguns consumidores, ao perceberem que suas reclamações estão sendo atendidas pela empresa ou ao receberem um pedido de desculpas, se sentem mais dispostos a utilizarem os serviços desta no futuro (TOMLINSON; MAYER, 2009). Por outro lado, de acordo com Wright et al.(1996), se a reclamação não for atendida de maneira satisfatória, os clientes mostram-se mais dispostos a recusar os serviços no futuro.

Wright et al. (1996) evidenciaram ainda que a percepção que o consumidor tem do problema, como estável e controlável, são determinantes para as intenções de recompra do consumidor. Consumidores desejam evitar a empresa no futuro quando percebem o problema como estável, enquanto que os consumidores que percebem que a empresa poderia ter feito algo para evitar o problema, provavelmente sentem raiva, ficam mais insatisfeitos e podem nunca mais comprar nesta empresa. (MAGNINI et al., 2007; HUANG, 2008; IGLESIAS, 2009)

Adicionalmente, a literatura sobre as emoções dos consumidores indica que quando os consumidores ficam com raiva, eles são mais propensos à ruptura da relação comercial, à reclamação, à mudança para concorrentes e a fazer um boca-a-boca negativo (BAGOZZI et al., 1999 , BECHWATI; MORRIN, 2003). Para Weiner (2000), as emoções são mediadoras entre as dimensões da Teoria da Atribuição e a predisposição do consumidor em renovar relações comerciais.
Em resumo, a Teoria da Atribuição possui três hipóteses sobre as reações dos consumidores a renovar as relações comerciais após falhas no serviço.

H1: Quanto maior a percepção da estabilidade das causas, maiores as emoções negativas. (FOLKES, KOLETSKY; GRAHAM, 1987)

H2: Quanto maior a percepção da responsabilidade da organização, maiores as emoções negativas. (WEINER, 2000)

H3: Quanto maior a percepção de emoções negativas, menor a predisposição do consumidor para renovar as relações comerciais. (FOLKES, KOLETSKY; GRAHAM, 1987 WEINER, 2000)

As relações postuladas nessas previsões estão representadas no modelo analítico da pesquisa, ilustrado na Figura 1.

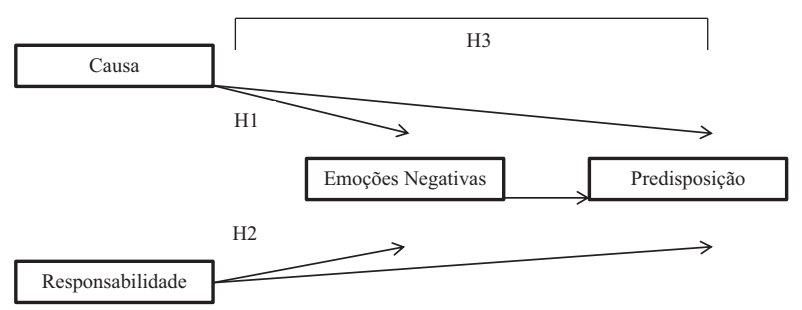

Figura 1: Modelo analítico do processo de finalização de relacionamentos comerciais com base na Teoria da Atribuição Fonte: Elaborado pelos autores deste artigo

A seção seguinte explora os procedimentos metodológicos da pesquisa.

\section{Procedimentos Metodológicos}

Esta seção está organizada em três subseções. Primeiramente é feita uma caracterização da natureza da pesquisa e da amostra utilizada, indicando os critérios para participação na pesquisa. Em seguida, serão apresentados o instrumento de pesquisa $e$ a forma de mensuração dos construtos do modelo analítico. Finalmente, a forma como os dados foram tratados no modelo de equação estrutural, incluindo a mediação, é identificada.

\subsection{Classificação da Pesquisa e da Amostra de Estudo}

A presente pesquisa é caracterizada como descritiva à medida que se propõe a descrever as carac- 
terísticas de um grupo de consumidores no que diz respeito a um conjunto de variáveis e suas relações. O modelo conceitual proposto na Figura 1 foi testado em um estudo de campo envolvendo 245 consumidores. Os participantes foram abordados aleatoriamente em shopping centers e supermercados.

Como abordagem inicial da coleta de dados, os participantes foram solicitados a indicar um prestador de serviço com o qual tivessem rompido o relacionamento nos últimos 12 meses. Tal restrição baseou-se em uma orientação de Weiner (2000) que indica que os consumidores podem ainda se lembrar de fatos a partir de uma relação comercial finalizada dentro deste período. Também solicitou-se aos respondentes que indicassem o tempo de suas relações, a principal motivação para o início das reflexões e qual o motivo foi determinante para o término do relacionamento comercial com o prestador de serviço.

\subsection{Instrumento de Pesquisa e Teste das Escalas}

Em razão da inexistência de escalas de medição das dimensões da Teoria da Atribuição para a ruptura das relações comerciais, foram desenvolvidas escalas com base nos procedimentos da Phase Completion Scales (HODGE; GILLESPIE, 2007). Essa escala consiste em uma frase seguida de reações de 11 pontos, que vão desde "certamente não" até "certamente sim". Segundo os autores, essa escala tende a aumentar a confiabilidade e a validade relativa quando comparada à de Likert.

O Quadro 1 apresenta as afirmações relativas a cada um das escalas desenvolvidas com a indicação da teoria que serviu de base para a sua construção. Com isto, as escalas da causa, responsabilidade, emoção e predisposição foram desenvolvidas e testadas. Os softwares SPSS e AMOS foram utilizados na análise. Um pré-teste do instrumento foi realizado com 20 participantes.

\begin{tabular}{|c|c|c|}
\hline & ITENS & BASE TEÓRICA \\
\hline \multirow{3}{*}{ Causa } & Você acha que o motivo determinante (a gota d'água) continua ocorrendo? & \multirow{3}{*}{$\begin{array}{l}\text { Folkes, Koletsky e } \\
\text { Graham (1987) }\end{array}$} \\
\hline & O motivo era temporário (não costumava acontecer)? & \\
\hline & Você acredita que o motivo poderia ocorrer novamente? & \\
\hline \multirow{4}{*}{ Responsabilidade } & A empresa/prestador de serviço poderia resolver o problema que ocasionou sua saída? & \multirow{4}{*}{ Weiner (2000) } \\
\hline & O motivo era de responsabilidade somente do prestador de serviço? & \\
\hline & $\begin{array}{l}\text { O motivo era algo que a empresa/prestador de serviço } \\
\text { teria controle para que não acontecesse? }\end{array}$ & \\
\hline & O motivo era controlável pela empresa/prestador de serviço? & \\
\hline \multirow{5}{*}{$\begin{array}{l}\text { Emoções } \\
\text { Negativas }\end{array}$} & Você sentiu raiva da empresa/prestador de serviço? & \multirow{5}{*}{ Weiner (2000) } \\
\hline & Você se sentiu enganado (a) pela empresa/prestador de serviço? & \\
\hline & No momento em que a situação ocorreu você ficou irritado (a)? & \\
\hline & Atualmente, você se sente aborrecido (a) com a empresa/prestador de serviços? & \\
\hline & Você se sentiu frustrado (a) com a finalização do relacionamento? & \\
\hline \multirow{6}{*}{$\begin{array}{l}\text { Predisposição } \\
\text { ao retorno }\end{array}$} & $\begin{array}{l}\text { Você voltaria a ser cliente desta empresa/prestador de serviço mesmo } \\
\text { diante de outras opções de mercado dos concorrentes? }\end{array}$ & \multirow{6}{*}{$\begin{array}{c}\text { Folkes, Koletsky e } \\
\text { Graham (1987) } \\
\text { Weine ( 2000) }\end{array}$} \\
\hline & Você recomendaria a um (a) amigo (a) os serviços desta empresa/prestador de serviço? & \\
\hline & Você se incomodaria de restabelecer a relação com esta empresa/prestador de serviço? & \\
\hline & $\begin{array}{l}\text { Por tudo o que aconteceu, é improvável que você retorne o } \\
\text { relacionamento com esta empresa/prestador de serviço? }\end{array}$ & \\
\hline & Você consideraria seriamente a possibilidade de retomar o relacionamento? & \\
\hline & Você evita utilizar quaisquer serviços oferecidos por esta empresa/prestador de serviço? & \\
\hline
\end{tabular}

Quadro 1: Escalas e Bases Teóricas

Fonte: Elaborado pelos autores deste artigo 
Para avaliar a confiabilidade das escalas foi utilizada a confiabilidade composta (CR), considerada satisfatória quando CR > 0,7 (FORNELL; LARKER, 1981). Para avaliar a validade convergente, foi utilizada a variância média extraída (AVE), considerada satisfatória quando AVE > 0,5 (GARVER; MENTZER, 1999). Por último, foi avaliada a validade discriminante, quando se verificou a máxima variância quadrada compartilhada (MSV) e a variância quadrada média compartilhada (ASV). (DeVELLIS, 1991; HAIR et al., 2006)

A análise fatorial confirmatória foi conduzida para avaliar a validade de construto e a confiabilidade das medidas observáveis (ANDERSON; GERBING, 1988; HAIR et al., 2006; SCHUMACKER; LOMAX, 2004).

\subsection{Estimação e Teste do Modelo Estrutural e Teste de Mediação}

Um modelo de mensuração foi estimado simultaneamente para todas as medidas utilizadas na pesquisa. Inicialmente, foi feita a aferição da multicolinearidade no intuito de analisar se variáveis que parecem distintas medem, na verdade, a mesma coisa. A avaliação da multicolinearidade foi feita através da análise do valor da tolerância e de seu inverso, o fator de inflação da tolerância (Variance Inflator Factor - VIF).

Hair et al. (2006) definem o VIF como o indicador do efeito que as outras variáveis independentes têm sobre o erro padrão de um coeficiente de regressão. Valores de tolerância menores que $10 \%$ e da VIF maiores que 10 indicam multicolinearidade (HAIR et al., 2006). Por último, foi realizada a análise da matriz de correlações para verificar a multicolinearidade das variáveis com o objetivo de verificar a existência de correlações maiores que 0,80 , o que indicaria multicolinearidade. (KLINE, 1998)

Em seguida foram utilizadas regressões múltiplas para testar os efeitos diretos das Hipóteses $\mathrm{H} 1$ e H2 (BARON; KENNY, 1986). Para testar os efeitos de mediação na hipótese $\mathrm{H} 3$, foram utilizados os procedimentos dos passos causais da mediação proposto por Baron e Kenny (1986), por meio da inclusão de cada variável mediadora separadamente em um modelo de regressão para cada variável dependente.

As técnicas indicadas por Cheung e Lau (2008), Hair et al. (2010), Preacher e Hayes (2004 e 2008) e Preacher, Rucker e Hayes (2007) foram usadas para analisar se as emoções negativas são mediadoras entre as dimensões da Teoria da Atribuição e a Predisposição. A diferença entre as duas técnicas é pelo fato de a primeira utilizar todo o modelo estrutural proposto por meio do software AMOS e a segunda se utiliza do módulo MEDIATE de Hayes e Preacher (2011) aplicado ao SPSS.

Na primeira técnica, o primeiro passo foi verificar o efeito direto e o nível de significância dessa relação sem as variáveis mediadoras no modelo estrutural. O segundo passo foi a inclusão das relações mediadoras entre as variáveis no modelo estrutural $e$ a indicação do bootstrapping de 2.000 reamostras com o intervalo de confiança em $95 \%$. A técnica para verificar o intervalo de confiança entre esse efeito mediador foi o bias-corrected method ou $B C$ method. Essa técnica consiste em fazer com que a distribuição não tenha viés de problemas com normalidade na construção de estimadores. (MOONEY; DUVAL, 1993)

\section{Resultados}

Esta seção está organizada em duas subseções. Primeiramente é feita uma caracterização socioeconômica da amostra e apresentados os testes das escalas. Em seguida são procedidos os testes do modelo estrutural e a discussão dos resultados.

\subsection{Análise Socioeconômica e Teste Inicial das Escalas}

Uma caracterização inicial do perfil da amostra indica que a amostra era relativamente homogênea em termos de participação de homens (45\%) e mulheres (55\%). A maior parte dos respondentes tinha entre 21 e 30 anos (58\%), e possuíam graduação completa (65\%). Empresas de setores variados foram indicadas pelos respondentes, sendo que a maioria estava ligada à cartões de crédito e à telefonia móvel celular (47\%), academia de ginástica (22\%) e salão de beleza (16\%). Os demais $15 \%$ são atribuídos para serviços de hotel/ pousada, oficina mecânica, restaurante dentre outros.

Em relação ao tempo de relacionamento, $41 \%$ dos respondentes indicaram que possuíam até um ano de relacionamento, $19 \%$ entre 1 e 2 anos, $16 \%$ entre 2 e 3 anos e $24 \%$, acima de 4 anos de relacionamento 
com o prestador de serviços. Especificamente sobre a motivação que fizeram com que os respondentes começassem a pensar na finalização do relacionamento comercial, $22 \%$ não estavam satisfeitos com o serviço, $22 \%$ indicaram que foi por causa da perda de qualidade na prestação dos serviços, $20 \%$ encontraram melhores opções oferecidas pelos concorrentes $15 \%$ foram por aspectos financeiros, $9 \%$ foram por negligência de empregados e $12 \%$ por outros motivos.

Resultados semelhantes foram encontrados sobre o motivo determinante que os respondentes decidiram finalizar o relacionamento com o prestador de serviço. Isso significa que os motivos iniciais preponderantes para que eles iniciassem foram também determinantes na finalização. Tal fato indica que as empresas não estão considerando a possibilidade do atendimento às reclamações como um bom antecedente da manutenção de um relacionamento. (TOMLINSON; MAYER, 2009; WRIGHT et al., 1996)

A Tabela 1 apresenta os testes iniciais para as escalas desenvolvidas neste estudo. Como pode-se notar, todas as cargas fatoriais para a construção das escalas de percepção da causa, percepção de responsabilidade, emoções negativas e predisposição ao retorno foram significativas e acima de 0,60, com Alpha de Cronbach maior que 0,8 .

Tabela 1: Propriedades de Medida do Modelo

\begin{tabular}{|c|c|c|}
\hline Construtos/ITEMS & $\begin{array}{c}\text { CARGAS } \\
\text { Fatoriais }\end{array}$ & $\begin{array}{c}\text { AlPHA DE } \\
\text { CRONBACH }\end{array}$ \\
\hline Causa & & 0,887 \\
\hline Ca1 & 0,889 & \\
\hline Ca2 & 0,889 & \\
\hline Ca3 & 0,869 & \\
\hline Responsabilidade & & \\
\hline Rp1 & 0,758 & \\
\hline Rp2 & 0,705 & \\
\hline Rp3 & 0,780 & \\
\hline Rp4 & 0,749 & \\
\hline Emoções Negativas & \\
\hline Em1 & 0,696 & 0,886 \\
\hline Em2 & 0,769 & \\
\hline Em3 & 0,750 & \\
\hline Em4 & 0,794 & \\
\hline
\end{tabular}

\begin{tabular}{|c|c|c|}
\hline Construtos/ITEMS & $\begin{array}{c}\text { CARgas } \\
\text { FAtoRIAIs }\end{array}$ & $\begin{array}{c}\text { AlPHA DE } \\
\text { CronBACH }\end{array}$ \\
\hline Em5 & 0,746 & \\
\hline Predisposição & & 0,869 \\
\hline Pr1 & 0,824 & \\
\hline Pr2 & 0,759 & \\
\hline Pr3 & 0,677 & \\
\hline Pr4 & 0,639 & \\
\hline Pr5 & 0,780 & \\
\hline Pr6 & 0,660 & \\
\hline
\end{tabular}

Nota: Índices de ajustamento estatístico: $\mathrm{X}^{2}=270,641, \mathrm{gl}=146, \mathrm{X}^{2} / \mathrm{gl}=1,85$, $\mathrm{IFI}=0,941, \mathrm{NNFI}=0,922, \mathrm{CFI}=0,940$ e RMSEA $=0,059$. De acordo com as indicações de Tabachnick e Fidell (2001) os seguintes índices são satisfatórios: $\mathrm{X}^{2} / \mathrm{gl}<3$; IFI, TLI e CFI > 0,90; e RMSEA < 0,08.

Fonte: Análise de dados

A próxima etapa de preparação no uso da técnica de modelagem em equações estruturais foi a verificação da confiabilidade e da validade convergente e discriminante dos construtos, por meio dos procedimentos de Fornell e Larker (1981) e de Garver e Mentzer (1999). Assim, foram realizadas as verificações por meio dos modelos de mensuração com consumidores brasileiros e canadenses.

Os resultados apresentados na Tabela 2 foram também satisfatórios no que se refere à confiabilidade e validade convergente e discriminante por meio das métricas da confiabilidade composta $(\mathrm{CR})$, variância média extraída (AVE), máxima variância quadrada compartilhada (MSV) e variância quadrada média compartilhada (ASV).

Tabela 2: Propriedades da confiabilidade e da validade convergente e discriminante

\begin{tabular}{|c|c|c|c||c|}
\hline Construtos & CR & AVE & MSV & ASV \\
\hline Responsabilidade & 0,835 & 0,507 & 0,521 & 0,352 \\
\hline Causa & 0,885 & 0,563 & 0,498 & 0,447 \\
\hline Predisposição & 0,870 & 0,529 & 0,441 & 0,299 \\
\hline Emoção & 0,887 & 0,611 & 0,521 & 0,420 \\
\hline
\end{tabular}

Nota: Indicações satisfatórias segundo Fornell e Larker (1981) e Garver e Mentzer (1999): CR > 0,7; AVE > 0,5; CR > AVE; MSV < AVE e ASV < AVE. As correlações foram satisfatórias segundo Tabachnick e Fidell (2001) $<0,80$.

Fonte: Análise de dados 


\subsection{Teste do Modelo Estrutural e Teste de Mediação}

Conforme indicação dos procedimentos metodológicos a serem utilizadas nesta segunda fase de análise dos dados, foram analisados os efeitos de multicolinearidade dos construtos testados na pesquisa. Os resultados apresentados na Tabela 3 sugerem que esse não é um problema nos dados levantados. (TABACHNIK; FIDELL, 2001)

Tabela 3: Estatística Descritiva e Correlações Bivariadas

\begin{tabular}{|c|c|c|c|c|c|c|}
\hline VARIÁVEL & MéDIA & DesuPad & $\mathbf{1}$ & 2 & 3 & 4 \\
\hline 1. Causa & 7,84 & 1,97 & $(0,887)$ & & & \\
\hline 2. Responsabilidade & 6,93 & 2,74 & $0,27^{*}$ & $(0,818)$ & & \\
\hline 3. Emoções Negativas & 5,83 & 2,93 & $0,163^{* *}$ & $0,669^{* *}$ & $(0,886)$ & \\
\hline 4. Predisposição & 6,15 & 2,68 & $0,108^{* *}$ & $0,444^{* *}$ & $0,664^{* *}$ & $(0,869)$ \\
\hline
\end{tabular}

Nota: Alpha de Cronbach para *p $<0.05$; e para **p $<0.001$.

Fonte: Análise de dados

A Tabela 4 mostra os resultados para as relações diretas entre as emoções negativas e a causa e a responsabilidade. De acordo com a H1 e a H2, as emoções negativas são significativamente relacionadas com a causa $(b=0,222, p<0,01)$ e com a responsabilidade $(b=0,830, p<0,001)$.

Tabela 4: Coeficientes das Regressões dos Efeitos Diretos das Emoções Negativas

\begin{tabular}{|c|c|c|c|c|c|c|c|}
\hline \multicolumn{2}{|c|}{ Diagrama dos Caminhos } & \multicolumn{2}{|c|}{$\begin{array}{l}\text { Cargas Não } \\
\text { Padronizadas }\end{array}$} & \multirow[b]{2}{*}{ Cargas Padronizadas ( $(\beta)$} & \multirow[b]{2}{*}{ Teste $T$} & \multicolumn{2}{|c|}{ HIPÓTESES } \\
\hline $\begin{array}{c}\text { Variável } \\
\text { Dependente } \\
{\left[\mathrm{R}^{2}\right]}\end{array}$ & Variável Independente & $\begin{array}{c}\text { Cargas } \\
\text { (b) }\end{array}$ & $\begin{array}{c}\text { Erros- } \\
\text {-padrão } \\
(\varepsilon)\end{array}$ & & & $\mathrm{p}$ & \\
\hline \multirow{2}{*}{$\begin{array}{c}\text { Emoções } \\
\text { Negativas } \\
{[0,474]}\end{array}$} & Causa & 0,222 & 0,083 & 0,163 & 2,680 & 0,007 & $\mathrm{H} 1$ \\
\hline & Responsabilidade & 0,830 & 0,115 & 0,669 & 7,204 & 0,000 & $\mathrm{H} 2$ \\
\hline
\end{tabular}

Fonte: Análise de dados

Para o teste de mediação, é necessário obter as seguintes condições: (1) as emoções negativas são antecedentes significantes da predisposição; (2) quando avaliadas separadamente, a causa e a responsabilidade são antecedentes significantes da predisposição; e (3) a relação entre a causa e a responsabilidade não deve ser significante ou deve ser fraca quando as emoções negativas são incluídas como antecedente nos modelos. Como demonstrado na Tabela 5 a seguir, as emoções negativas, a causa e a responsabilidade são antecedentes significantes da predisposição. Assim, as condições 1 e 2 estão satisfeitas.

Tabela 5: Coeficientes da Regressão dos Efeitos Diretos da Predisposição

\begin{tabular}{|c|c|c|c|c|c|c|}
\hline \multicolumn{2}{|c|}{ Diagrama dos Caminhos } & \multicolumn{2}{|c|}{ Cargas Não Padronizadas } & \multirow{2}{*}{$\begin{array}{c}\text { Cargas } \\
\text { Padronizadas ( }(\beta)\end{array}$} & \multirow{2}{*}{ Teste $T$} & \multirow{2}{*}{$\mathbf{P}$} \\
\hline Variável Dependente $\left[\mathrm{R}^{2}\right]$ & Variável Independente & Cargas $(\mathbf{b})$ & Erro-padrão $(\varepsilon)$ & & & \\
\hline \multirow{2}{*}{$\begin{array}{c}\text { Predisposição } \\
{[0,252]}\end{array}$} & Causa & 0,262 & 0,090 & 0,203 & 2,901 & 0,004 \\
\hline & Responsabilidade & 0,536 & 0,097 & 0,460 & 5,510 & 0,000 \\
\hline $\begin{array}{c}\text { Predisposição } \\
{[0,442]}\end{array}$ & Emoções Negativas & 0,603 & 0,071 & 0,665 & 8,501 & 0,000 \\
\hline
\end{tabular}

Fonte: Análise de dados 
De acordo com os resultados apresentados na TAB. 6, quando todas as variáveis estão relacionadas em conjunto e a causa, a responsabilidade e as emoções negativas estão relacionadas como antecedentes da predisposição. Nesse sentido, foi verificado que a causa $e$ a responsabilidade não são antecedentes significantes de predisposição (Causa $\rightarrow$ Predisposição: $b=$ $0,14, p=0,080$ e Responsabilidade $\rightarrow$ Predisposição: $\mathrm{b}=0,094, \mathrm{p}=0,374)$. As emoções negativas são os únicos antecedentes significantes da predisposição (emoções negativas: $b=0,525, p<0,001$ ).

Finalmente, a condição 3 foi também estabelecida quando foi comparado o efeito direto da causa e responsabilidade com a predisposição e quando se incluiu as emoções negativas como mediadora entre a Causa/Responsabilidade e a Predisposição a fim de também entender seus efeitos indiretos.

Tabela 6: Coeficientes da Regressão dos Efeitos Diretos e Indiretos da Predisposição - Todas as Variáveis

\begin{tabular}{|c|c|c|c|c|c|c|}
\hline \multicolumn{2}{|c|}{ Diagrama dos Caminhos } & \multicolumn{2}{|c|}{ CARgas Não Padronizadas } & \multirow{2}{*}{$\begin{array}{c}\text { Cargas } \\
\text { Padronizadas ( }(\beta)\end{array}$} & \multirow{2}{*}{ Teste $T$} & \multirow{2}{*}{$\mathbf{P}$} \\
\hline Variável Dependente $\left[\mathrm{R}^{2}\right]$ & Variável Independente & Cargas $(\mathbf{b})$ & Erro-padrão $(\varepsilon)$ & & & \\
\hline \multirow{2}{*}{$\begin{array}{c}\text { Emoções } \\
\text { Negativas } \\
{[0,465]}\end{array}$} & Causa & 0,207 & 0,084 & 0,151 & 2,475 & 0,013 \\
\hline & Responsabilidade & 0,826 & 0,115 & 0,665 & 7,154 & 0,000 \\
\hline \multirow{3}{*}{$\begin{array}{c}\text { Predisposição } \\
{[0.431]}\end{array}$} & Causa & 0,140 & 0,080 & 0,112 & 1,751 & 0,080 \\
\hline & Responsabilidade & 0,094 & 0,105 & 0,083 & 0,889 & 0,374 \\
\hline & $\begin{array}{l}\text { Emoções } \\
\text { Negativas }\end{array}$ & 0,525 & 0,092 & 0,574 & 5,733 & 0,000 \\
\hline
\end{tabular}

Fonte: Análise de dados

Assim, a Tabela 7 apresenta os efeitos diretos com e sem a variável mediadora e os efeitos indiretos são indicados.

Tabela 7: Análise dos tipos de mediação no modelo estrutural

\begin{tabular}{|c|c|c|c|c|c|c|c|}
\hline $\begin{array}{c}\text { RELACIONAMENTO } \\
\text { DAS VARIÁveIS }\end{array}$ & \multicolumn{6}{|c|}{$\begin{array}{c}\text { Efeitos DiRetos } \\
\text { com Emoções } \\
\text { Negativas } \\
\text { (VARIÁvel } \\
\text { MediadoRA) }\end{array}$} & \multirow[t]{2}{*}{ Tipo de Mediação Observada } \\
\hline $\begin{array}{c}\text { Boot- } \\
\text { Strapping } \\
\text { com } 2.000 \text { reamostras }\end{array}$ & $\begin{array}{c}\text { Cargas } \\
\text { Padronizadas }\end{array}$ & Sig. & $\begin{array}{c}\text { Cargas } \\
\text { Padronizadas }\end{array}$ & Sig. & $\begin{array}{c}\text { Cargas } \\
\text { Padronizadas }\end{array}$ & Sig. & \\
\hline Causa & 0,163 & $0,007^{* *}$ & $-0,131$ & 0,457 & 0,257 & $0,001^{* *}$ & Mediação Parcial \\
\hline Responsabilidade & 0,669 & $0,000^{* * * * *}$ & $-0,18$ & 0,099 & 0,19 & $0,009^{* * *}$ & Mediação Parcial \\
\hline
\end{tabular}

Nota: *** $\mathrm{p}<0,001 e^{* *} \mathrm{p}<0,01$.

Fonte: Análise de dados

Foi percebido que, por meio da técnica de bootstrapping na modelagem em equações estruturais e do $B C$ method, foi possível não apenas verificar os efeitos da me- diação das emoções negativas, mas também foi possível preencher a lacuna deixada por Weiner (2000), no que se refere ao tipo de mediação, no caso mediação parcial. 
Em seguida, foi utilizada a técnica de Preacher e Hayes (2004 e 2008) e Preacher, Rucker e Hayes (2007), para a verificação do efeito de mediação. Para isso, foi indicada a Predisposição como a variável dependente (VD), a Causa e a Responsabilidade como variáveis independentes (VI) e as Emoções Negativas como variável mediadora (Med). Assim, o efeito direto (B) da Causa com a Predisposição foi igual a 0,2410 com $\mathrm{t}=2,9871$ e $\mathrm{p}<0,0031$ e o efeito direto da Responsabilidade com a Predisposição foi igual a 0,3699 com $\mathrm{t}=6,3919$ e $\mathrm{p}<0,0000$, ficando o modelo com $\mathrm{R}^{2}=0,2119, \mathrm{~F}=32,5401$ e $\mathrm{p}<0,001$.
O segundo passo foi verificar os efeitos diretos das variáveis indiretas com a variável mediadora. Assim, o efeito direto (B) da Causa com as Emoções Negativas foi igual a $0,1930 \mathrm{com} t=2,4350$ e $\mathrm{p}<0,0156$ e o efeito direto da Responsabilidade com as Emoções Negativas foi igual a 0,5968 com $\mathrm{t}=10,4998$ e $\mathrm{p}<0,0000$, ficando o modelo com $R^{2}=0,3676, F=70,3315$ e $p<0,001$.

O terceiro e último passo é incluir as variáveis mediadoras no modelo juntamente com a variável independente, no intuito de verificar o efeito (B) do coeficiente. Assim, é mostrada a Tabela 8, que apresenta os efeitos com todas variáveis no modelo.

Tabela 8: Efeito de mediação das emoções negativas entre as dimensões da Teoria da Atribuição (causa e responsabilidade) e a predisposição

\begin{tabular}{|c|c|c|c|c|}
\hline \multicolumn{1}{|c|}{ VARIÁveIS } & EFEITO (B) & ERRO PADRÃo & T & P \\
\hline Emoções Negativas & 0,4359 & 0,0593 & 7,3555 & $* * *$ \\
\hline Causa & 0,1569 & 0,0740 & 2,1214 & 0,0349 \\
\hline Responsabilidade & 0,1097 & 0,0632 & 1,7355 & 0,0839 \\
\hline
\end{tabular}

Nota: Estatísticas do modelo: $\mathrm{R}^{2}=0,0448, \mathrm{~F}=36,0851$ e $\mathrm{p}<0,001$. *** $\mathrm{p}<0,001$.

Fonte: Análise de dados

Para inferir se há efeito de mediação, o efeito $(\Omega)$ tende a ficar mais fraco ou insignificante, quando indicado com todas as variáveis mediadoras no modelo. Assim, percebe-se que os efeitos eram significantes com a relação direta entre as variáveis independentes (Causa e Responsabilidade) e a variável dependente (Predisposição) e quando se incluiu a variável mediadora, houve redução no efeito ou se tornou insignificante em todas as relações. A Predisposição $\leftarrow$ Causa, passa de 0,2410 ( $\mathrm{p}=0,0031)$ para 0,1569 $(\mathrm{p}=0,0349)$ e a Predisposição $\leftarrow$ Responsabilidade, passa de 0,3699 $(\mathrm{p}<0001)$ para 0,1097 ( $\mathrm{p}=0,0839)$.

Portanto, por meio das duas técnicas adotadas para a verificação dos efeitos de mediação das Emoções Negativas entre as Dimensões da Teoria da Atribuição (Causa e Responsabilidade) e a Predisposição, foi possível não somente entender que as Emoções Negativas é variável mediadora na relação entre Causa/Responsabilidade e a Predisposição, mas também indicar o tipo de mediação, verificada como mediação parcial. Isso significa que há efeito significante não apenas entre as variáveis independentes (Causa/Responsabilidade) com a variável dependente (Predisposição), mas também entre a variável mediadora (Emoções Negativas) com a variável dependente.

Esses achados reforçam os textos de Weiner (2000), Folkes, Koletsky e Graham (1987) e Folkes
(1984) de que a percepção da causa como estável torna menor a predisposição ao retorno do consumidor ao relacionamento comercial finalizado. Os resultados também estão em linhas com as pesquisas de Weiner, Graham e Chandler (1982), ao evidenciarem que quanto maior a estabilidade da causa, maior será a intensidade de sentimentos negativos como a raiva (FOLKES; KOLETSKY; GRAHAM, 1987). Diferentemente disso, quando o consumidor tem uma percepção de que as causas são temporárias, os sentimentos se tornam menos intensos (FOLKES; KOLETSKY; GRAHAM, 1987; BADOVICK; HADAWAY; KAMINSKI, 1992)

Já quanto ao construto Responsabilidade, Folkes (1984), Weiner (2000) e Farias, Góis e Oliveira (2000) evidenciavam que a responsabilidade externa influencia na progressão das emoções e pode impactar na saída do relacionamento. O lócus da causa, a percepção de responsabilidade por parte da empresa também influencia nas reações afetivas à performance e a progressão de emoções que podem ser atenuadas com a combinação desses fatores (FOLKES, 1984). Culpa e embaraço são ligados à dimensão do lócus da causa. Essas reações afetivas influenciam subsequentes decisões sobre o produto ou serviço. (WEINER, 2000)

Conforme Folkes (1984), quando a falha do produto ou serviço é percebida de responsabilidade da 
empresa há uma desigualdade na relação de troca entre comprador e prestador de serviço e, portanto, o consumidor se sente enganado. No estudo de Farias, Góis e Oliveira (2000) evidencia-se que a responsabilidade externa pode impactar na saída do relacionamento.

Para a variável mediadora Emoções Negativas, há o reforço do estudo teórico de Folkes (1984), Folkes, Koletsky e Graham (1987), Weiner (2000) que evidenciaram que as emoções negativas levam o consumidor a uma menor disposição a utilizar novamente os serviços da empresa/prestador de serviços.

Finalmente, o modelo proposto foi testado de acordo com a Figura 1 anteriormente apresentada. A Tabela 9 é composta pelos dois antecedentes das emoções negativas (Causa: $b=0,222, p=0,008$ e Responsabilidade: $\mathrm{b}=0,830, \mathrm{p}<0,001)$. Ressalta-se que as emoções negativas são os únicos antecedentes da predisposição (emoções negativas: $b=0,612, p<$ $0,001)$. Assim, há os seguintes resultados dos índices de ajuste estatístico do modelo (proposto): $\mathrm{X}^{2}=245,878$; comparative fit index $(\mathrm{CFI})=0,951$; normed fit index $(\mathrm{NFI})=0,901$; incremental fit index $(\mathrm{IFI})=0,951$; non-normed fit index (NNFI) = 0,936; e root mean square error of approximation (RMSEA) $=0,059$ (com intervalo de confiança de $90 \%$ [IC] entre 0,048-0,071). De acordo com a H3, as emoções negativas estão relacionadas de modo significante à predisposição e é mediadora entre a Causa/Responsabilidade e a predisposição. Portanto, o modelo proposto (resultados finais na Figura 2) é consistente, foi testado empiricamente e todos os resultados dos índices de ajuste estatístico produzidos neste modelo estrutural são considerados satisfatórios. (HAIR et al., 2006)

Tabela 9: Coeficientes da Regressão dos Efeitos Diretos e Indiretos da Predisposição - Modelo Proposto

\begin{tabular}{|c|c|c|c|c|c|c|c|}
\hline \multicolumn{2}{|c|}{ Diagrama dos Caminhos } & \multicolumn{2}{|c|}{ CARgas Não Padronizadas } & \multirow{2}{*}{$\begin{array}{c}\text { Cargas } \\
\text { Padronizadas } \\
\text { (ß) }\end{array}$} & \multirow[b]{2}{*}{ Teste $T$} & \multirow[b]{2}{*}{$\mathbf{P}$} & \multirow[b]{2}{*}{ HiPÓTESES } \\
\hline $\begin{array}{c}\text { Variável De- } \\
\text { pendente } \\
{\left[\mathrm{R}^{2}\right]}\end{array}$ & Variável Independente & $\begin{array}{c}\text { Cargas } \\
(\mathbf{b})\end{array}$ & $\begin{array}{l}\text { Erro-padrão } \\
(\varepsilon)\end{array}$ & & & & \\
\hline \multirow{2}{*}{$\begin{array}{c}\text { Emoções } \\
\text { Negativas } \\
{[0,474]}\end{array}$} & Causa & 0,222 & 0,083 & 0,163 & 2,680 & 0,008 & $\mathrm{H} 1$ \\
\hline & Responsabilidade & 0,830 & 0,115 & 0,669 & 7,204 & 0,000 & $\mathrm{H} 2$ \\
\hline $\begin{array}{c}\text { Predisposição } \\
{[0,441]}\end{array}$ & Emoções Negativas & 0,612 & 0,071 & 0,664 & 8,590 & 0,000 & $\mathrm{H} 3$ \\
\hline
\end{tabular}

Fonte: Análise de dados

A Figura 2 mostra os índices ajustados no modelo testado.
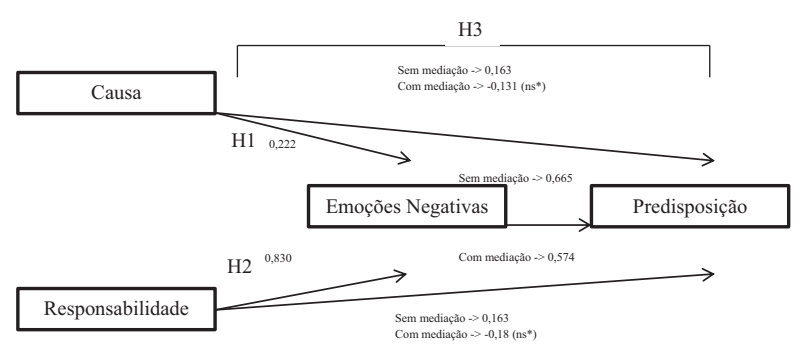

Nota: *Não significante.

Figura 2: Resultados finais dos coeficientes regressivos no modelo proposto do processo de finalização de relacionamentos comerciais por meio da Teoria da Atribuição.

Fonte: Elaborada pelos autores deste artigo

\section{Considerações Finais}

Este estudo analisou a predisposição dos consumidores a reiniciarem relações comerciais que foram rompidas no passado devido a questões de falhas em empresas prestadoras de serviços, com base na Teoria da Atribuição. Ao fazer isso, a pesquisa acrescenta um fundamento teórico, que demonstra a influência das construções atributivas de causa e responsabilidade nas variáveis consequentes - emoções negativas $e$ predisposição ao retorno.

Como contribuição metodológica inicial, destaca-se o desenvolvimento de novas medidas de mensuração para os construtos das dimensões da Teoria Atribuição e para a predisposição do consumidor ao 
retorno a um relacionamento comercial finalizado. Essas medidas podem ser utilizadas em estudos futuros que tratem sobre a temática.

Como implicações gerenciais, os resultados da amostra pesquisada indicam que grande parte dos motivos determinantes para o final do relacionamento foram os mesmos dos iniciais, o que sugere que: ou as empresas ou não tomaram conhecimento dos motivos iniciais da insatisfação dos consumidores ou, não tomaram providências para reverter às causas. Numa visão prática, isso contrasta com a importância que vem sendo dada à gestão do relacionamento com o cliente.

Nesse sentido, o estudo evidencia que há espaço para a ampliação de ações gerenciais de retenção de clientes, focadas na reversão de insatisfações iniciais, abordadas neste trabalho como motivos iniciais para o começo das ponderações do consumidor quanto a sair ou não do relacionamento, ou, de retorno de relações comerciais, voltadas para a solução dos motivos que foram determinantes para o fim da relação.

A pesquisa deixa claro, ainda, que as empresas precisam estar preparadas para lidar com um consumidor insatisfeito, evitando que esta insatisfação evolua para sentimentos negativos mais fortes, como a raiva já que, como comprovado por esta pesquisa, esses sentimentos diminuem a predisposição do consumidor em retornar ao relacionamento comercial finalizado.

Uma prática gerencial que pode ser adotada na tentativa de impedir que os motivos iniciais venham a se tornar motivos determinantes e com isso ampliar as intenções de retorno do consumidor ao relacionamento é a utilização das reclamações dos clientes como informação para a resolução de problemas existentes na organização. Ressalta-se que é importante tornar visível ao consumidor que as suas reclamações estão sendo atendidas pela empresa.

A alternativa que pode ser adotada pelas organizações seria utilizar bases de dados de reclamações de clientes como fonte de informação para a resolução de problemas existentes. Outra alternativa seria a realização de ações a partir de um estudo prévio do histórico do relacionamento terminado (gerenciamento do cliente perdido).

Em síntese, a empresa precisa assegurar para o consumidor que o motivo de sua insatisfação, que o fez sair do relacionamento comercial, foi identificado, analisado e resolvido, para que seja evidenciado o compromisso da empresa solucionar a insatisfação do cliente, visto que, a estabilidade da causa possui um efeito significante na predisposição do consumidor. Ou seja, ao perceber que a causa que o fez sair do relacionamento ainda existe, o consumidor tenderá a não retornar ao relacionamento comercial finalizado. Para tanto, faz-se necessário que as empresas mantenham bancos de dados sobre os seus clientes que incluam, além de um histórico de vendas, um histórico de reclamações e o registro do motivo que o fez sair.

Como limitações da pesquisa e direcionamentos para futuros estudos em torno desta temática, sugere-se que esta pesquisa seja replicada para que a escala proposta seja refinada. Em segundo lugar, embora tenha sido verificado que os consumidores brasileiros reagem emocionalmente em situações de falha do serviço, a importância deste construto como um mediador das reações entre outros grupos de consumidores em outras culturas é desconhecida. Neste sentido, seria interessante comparar com os resultados encontrados aqui com consumidores brasileiros. Assim, sugere-se replicar este estudo para outros tipos de serviço e em outros países, a fim de ampliar os horizontes da Teoria da Atribuição e com rupturas de serviços entre outras culturas.

\section{REFERÊNCIAS}

ANDERSON, J. C.; GERBING, D. W. Structural Equation Modeling in Practice: a review and recommended twostep approach. Psychological Bulletin, Washington DC, n. 103, p. 411-423, 1988.

BADOVICK, G. J.; HADAWAY, F. J.; KAMINSKI, P. F. Attributions and emotions: the effects on salesperson motivation after successful vs. unsuccessful quota performance. Journal of Personal Selling \& Sales Management, Münster, v. 12, n. 3, 1992.

BAGOZZI, R. P.; GOPINATH, M.; NYER, P. U. The Role of Emotions in Marketing. Journal of the Academy of Marketing Science, New York, v. 27, n. 2, p. 184-206, 1999.

BARON, R. M.; KENNY, D. A. The Moderator-Mediator Variable Distinction in Social Psychological Research: Conceptual, Strategic, and Statistical Considerations.

Journal of Personality and Social Psychology, Washington DC, v. 51, n. 6, p. 1.173-1.182, 1986. 
BECHWATI, N. N.; MORRIN, M. Outraged Consumers: Getting Even at the Expense of Getting a Good Deal.

Journal of Consumer Psychology, London, v. 13, n. 4, p. 440-453, 2003.

BERRY, L. Emerging perspectives on services marketing. American Marketing Association, Chicago, p. 25-28, 1983.

BITNER, M. J.; BOOMS, B. H.; TETREAULT, M. S. The service encounter: Diagnosing favorable and unfavorable incidents. Journal of Marketing, Chicago, v. 54, n. 1, p. 71-84, 1990.

BONIFIELD, C.; COLE, C. Affective responses to service failure: Anger, regret, and retaliatory versus conciliatory responses, Marketing Letters, New York, v. 18, n.1, p. 85-99, June, 2007.

BURNKRANT, R. E. Attribution theory in marketing research: problems and prospects. Advances in Consumer Research, Duluth, v. 2, n. 1, p. 465-470, 1975.

COULTER, R. A.; LIGAS, M. The long good-bye: The dissolution of customer-service provider relationship.

Psychology \& Marketing, Malden, v. 17, n. 8, p. 669695, 2000.

CURREN, M. T.; FOLKES, V. S. Attributional influences on consumers' desire to communicate about products .

Psychology and Marketing, Malden, v. 4, n. 1, p. 31-45, 1987.

DEVELLIS, R. F. Scale development: theory and applications. Newbury Park, CA: SAGE Publications, 1991.

DWYER, R.; SCHURR, P.; OH, S. Developing buyerseller relationships and networks. Journal of Marketing, Chicago, v. 29, n. 1, p. 11-27, 1987.

FOLKES, V. S.; KOETSKY, S.; GRAHAM, J. L. A field study of causal inferences and consumer reaction: The view from the airport. Journal of Consumer Research, Chicago, v. 13, n. 4, p. 534-539, 1987.

FOLKES, V. S.; KOETSKY, S.; GRAHAM, J. L. A field study of causal inferences and consumer reaction: The view from the airport. Journal of Consumer Research, Chicago, v. 13, n. 4, p. 534-539, 1987.
FOLKES, V. S.; KOETSKY, S.; GRAHAM, J. L. Recent attribution research in consumer behavior: A review and new directions. Journal of Consumer Research, Chicago, v. 14, n. 4, p. 548-565, 1988.

FORNELL, C.; LARKER, D. Evaluating Structural Equation Models with Unobservable Variables and Measurement Error. Journal of Marketing Research Chicago, v. 18, n.3, p. 39-50, 1981.

GARVER, Michael S.; MENTZER, John T. Logistics research methods: employing structural equation modeling to test for construct validity. Journal of Business

Logistics, Malden, v. 20, n. 1, p. 33-57, 1999.

GEDEON, I. M.; FEARNE, A.; POOLE, N. The role of inter-personal relationships in the dissolution of business relationships. Journal of Business \& Industrial Marketing, Bingley, v. 24, n. 3-4, p. 218-226, 2009.

GILLY, M. C.; GELB, B. D. Post-purchase consumer processes and the complaining consumer. Journal of Consumer Research, Chicago, v. 9, n. 3, p. 323-328, 1982.

GRÖNROOS, C. Quo Vadis, Marketing? Toward a Relationship Marketing Paradigm. Journal of Marketing Management, London, v. 10 p. 347-360. 1994.

HAIR, J. F.; BLACK, W. C.; BABIN, B. J., ANDERSON, R. E.; TATHAM, R. L. Multivariate Data Analysis, 6th Edition, Pearson Education Inc, Upper Saddle River: New Jersey, 2006.

HALINEN, A.; TÄHTINEN, J. Ending exchange relationships: what do we know about them? Proc. In: The 16th IMP International Conference, Bath, U.K., 2000.

HELLIER, P. K. et al. Customer repurchase intention a general structural equation model. European Journal of Marketing, Bingley, v. 37, n. 11/12, p. 1.762-1.800, 2003.

HEIDER, F. The psychology of interpersonal relations. New York: John Wiley \& Sons, 1958.

HODGE, D. R.; GILLESPIE, D. F. Phrase Completion Scales: a better measurement approach than likert scales? Journal of Social Service Research, London, v. 33, n. 4, p. 1-12, 2007. 
HUANG, W. H. The impact of other-customer failure on service satisfaction. International Journal of Service Industry Management, v. 19, v. 4, p. 521-536, 2008.

IGLESIAS, V. The attribution of service failures: effects on consumer satisfaction. The Service Industries Journal, v. 29, n. 2, p. 127-141, 2009.

JONES, E. E. et al. Attribution: perceiving the causes of Behavior, Morristown, NJ: General Learning Press, 1971.

KELLEY, H. Attribution Theory in Social Psychology. In: Nebraska Symposium of Motivation, v. 15, ed. D. Levine, Lincoln: University of Nebraska Press, p. 192-238, 1967.

MAGNINI, V. P. et al. The service recovery paradox: justifiable theory or smoldering myth? Journal of Services Marketing, Bingley, v. 21, n. 3, p. 213-225, 2007.

MICHALSKI, S. Types of customer relationship ending processes. Journal of Marketing Management, London, v. 20, n. 9, p. 977-999, 2004.

MIZERSKI, R. W.; GOLDEN, L. L.; KERNANT, J. B. The attribution process in consumer decision making. Journal of Consumer Research, Chicago, v. 6, n. 2, p. 123-140, 1979.

MORGAN, R.; HUNT, S. The commitment-trust theory of relationship marketing. Journal of Marketing, Chicago, v. 58, July, p. 20-38, 1994.

PRESSEY, A. D.; MATHEWS, B. P. Jumped, pushed or forgotten? Approaches to dissolution. Journal of Consumer Behavior, Malden, v. 19, n. 12, p. 131-155, 2003.

PRESSEY, A. D.; SELASSIE, H. Motives for dissolution in export relationships: evidence from the UK. Journal of Consumer Behavior, Malden, v. 6, n. 2-3, p. 132-145, 2007.

ROBICHEAUX, R.; COLEMAN, J. The Structure of Marketing Channel Relationships. Journal of the Academy of Marketing Science, New York, v. 22, n.1, p. 38-51, 1995.

ROOS, I.; STRANDVIK, T. Diagnosing the termination of customer relationships. American Marketing

Association Conference, Dublin, 1997.
SCHUMACKER, R. E.; LOMAX, R. G. A Beginner's Guide to Structural Equation Modeling, Lawrence Erlbaum Associates, Inc., Mahwah: New Jersey, 2004.

STEWART, K. The customer exit process: A review and research agenda. Journal of Marketing Management, London, v. 14, n. 4, p. 235-250, 1998.

TABACHNICK, B. G.; FIDELL, L. S. Using Multivariate Statistics, 4th Edition, Allyn and Bacon, Needham Heights: MA, 2001.

\section{TÄHTINEN, J. The dissolution process of a} business relationship. A case study from tailored software business. Faculty of Economics and Industrial Management, University of Oulu, Finland, Oulu, 2001.

TÄHTINEN, J.; HAVILA, V. Enhancing research in exchange relationship dissolution. Journal of Marketing Management, London, v. 20, n. 9, p. 919-926, 2004.

TOMLINSON, E. C.; MAYER, R. C. The role of causal attribution dimensions in trust repair. Academy of Management Review, New York, v. 34, n. 1, p. 85-104, 2009.

WEINER, B. Attributional thoughts about consumer behavior. Journal of Consumer Research, Chicago, v. 27, n. 3, p. 382-387, 2000.

WRIGHT, R. E. et al. Effects of dissatisfying experiences on repatronage intentions and negative word-of-mouth behavior of university students. Journal of Consumer Satisfaction, Dissatisfaction and Complaining Behavior, Las Vegas, v. 9, p. 221-228, 1996. 